\title{
Control of hydroxylation regioselectivity by hyoscyamine $6 \beta$-hydroxylase as revealed by crystallographic and QM/MM studies
}

\author{
Z. Wojdyla, A. Kluza, T. Borowski \\ Jerzy Haber Institute of Catalysis and Surface Chemistry, Polish Academy of Sciences, Niezapominajek 8, Krakow, Poland \\ zuzanna.wojdyla@ikifp.edu.pl
}

Hyoscyamine $6 \beta$-hydroxylase $(\mathrm{H} 6 \mathrm{H})$ is a bifunctional 2-oxoglutarate/Fe(II)-dependent dioxygenase that catalyzes the two final steps in the biosynthesis of scopolamine [1], that is a regioselective hydroxylation of hyoscyamine at the C6 position, followed by a formation of the epoxide ring utilising the installed hydroxy group [2]. The combination of crystallographic and computational studies on $\mathrm{H} 6 \mathrm{H}$ :hyoscyamine complex provided insight into the substrate binding and the selectivity of the enzymatic reaction [3].

The QM/MM studies reveal that the regioselectivity of the hydroxylation reaction is dictated by only a few residues (i.e. Lys-129, Tyr326, Lys-330), which promote the reaction occurring at the C6 site and at the same time hinder the alternative channel proceeding at the neighbouring (C7) position. Notably, the electronic properties of the reactants, that is hyoscyamine and the active site, do not favour any of the reaction channels, which suggests that switching regioselectivity of the oxygen rebound and thus obtaining other potentially useful alkaloids, may be achieved by targeting the residues in vicinity of the reactants.

[1] Hashimoto T., Matsuda J. \& and Y. Yamada Y. (1993), FEBS Lett. 329, 35-39

[2] Li L., Van Belkum M.J. \& Vederas J.C. (2012) Bioorg. Med. Chem. 20, 4356-4363

[3] Kluza A., Wojdyla Z., Mrugala B., et al. (2020) Dalton T. 49, 4454-4469

\section{Keywords: metalloenzymes; reaction mechanisms; reaction regioselectivity; computation}

This research project was supported by the National Science Centre, Poland and PL-Grid Infrastructure and in part by Project PROM - International scholarship exchange of PhD candidates and academic staff, financed by the European Social Fund implemented operational programme Knowledge Education Development, project: International scholarship exchange of PhD candidates and academic staff, contract number PPI/PRO/2019/1/00021/U/001. 